\title{
Assessment of functioning in patients with schizophrenia and schizoaffective disorder with the Mini-ICF-APP: a validation study in Italy
}

\author{
Federica Pinna ${ }^{1}$, Andrea Fiorillo ${ }^{2}$, Massimo Tusconi ${ }^{1}$, Beatrice Guiso ${ }^{1}$ and Bernardo Carpiniello ${ }^{1 *}$
}

\begin{abstract}
Background: The aim of the study was to evaluate validity of the Italian Mini-ICF-APP (Mini-ICF Rating for Limitations of Activities and Participation in Psychological Disorders) in schizophrenia and related disorders.

Methods: 74 outpatients affected by schizophrenia or schizoaffective disorders attending a University-based community mental health centre were recruited to the study. All participants underwent comprehensive evaluation using standardized instruments to assess clinical, neurocognitive and functional status. Concurrent validity of Mini-ICF-APP was evaluated and compared to severity scores obtained using the Clinical Global Impression-Schizophrenia scale (CGI-SCH), Positive and Negative Syndrome scale (PANSS), Mini Mental State Examination test (MMSE), Brief Assessment of Cognition in Schizophrenia scale (BACS) and Personal and Social Performance scale (PSP). Construct validity was evaluated by comparing scores obtained at Mini-ICF-APP by remitted versus non-remitted patients, and by recovered versus unrecovered patients. Discriminant validity was evaluated comparing scores on Mini-ICF-APP and Subjective Well-being (SWN) scale. Results: the total score and 12 out of the 13 Mini-ICF-APP items correlated significantly with total score at PSP; Mini-ICF-App total score was moreover significantly correlated with total scores at CGI-SCH, PANSS, MMSE, as well as with several BACS items. Total scores obtained at Mini-ICF-APP were significantly higher among remitted and recovered patients. No relevant correlations were found between scores of Mini-ICF-APP and SWN scales.
\end{abstract}

Results: The total score and 12 out of the 13 Mini-ICF-APP items correlated significantly with total score at PSP; MiniICF-App total score was moreover significantly correlated with total scores at CGI-SCH, PANSS, MMSE, as well as with several BACS items. Total scores obtained at Mini-ICF-APP were significantly higher among remitted and recovered patients. No relevant correlations were found between scores of Mini-ICF-APP and SWN scales.

Conclusion: the Italian version of Mini-ICF-APP is a valid instrument for use in evaluating functioning in chronic patients with schizophrenia and related disorders.

Keywords: Mini-ICF-APP, Validity, Outpatients, Schizophrenia, Schizoaffective disorders

\section{Background}

Among all mental disorders, schizophrenia is undoubtedly the most frequently related to the development of disability [1]. Indeed, approx. $80 \%$ of adults affected by schizophrenia and related disorders have been estimated to show persistent deficits in personal and social functioning [2].

\footnotetext{
*Correspondence: bcarpini@iolit

1 Department of Public Health, Clinical and Molecular Medicine-Unit of Psychiatry, University of Cagliari, Via Liguria 13, 0917 Cagliari, Italy Full list of author information is available at the end of the article
}

Although clinical remission [3] is seen as the main goal of treatment, nowadays recovery, which implies a satisfactory level of functioning [4], is viewed as the ultimate target. Thus, the importance of personal and social functioning is of course indisputable, also bearing in mind that functioning deficits are comprised among the basic diagnostic criteria for schizophrenia and other mental disorders. However, in clinical practice the routine assessment of functioning is still uncommon for a series of reasons, likely including the lack of a simple, user-friendly rating 
scale for use as a reference tool; however, on the international scenario [5] a series of reliable and valid instruments such as GAF [6], SOFAS [7], PSP [8], and WHO-DAS2 [9] are available. In this context, the Mini-ICF-APP, specifically designed in line with parameters of the World Health Organization International Classification of Functioning, Disability and Health (ICF) for use in mental health settings, was first developed and validated in Germany [10, 11] and recently translated and validated in English [12] and Italian [13]. The original German version was validated on a sample of patients affected by non-psychotic mental disorders, whilst both the English and Italian versions were validated using mixed patient samples, including a limited number of subjects with schizophrenia or other psychotic disorders. Accordingly, the present study was designed with the aim of validating the instrument for use in schizophrenia and schizoaffective disorders, in the context of an ongoing observational, prospective study on remission and recovery in chronic psychotic patients [14].

\section{Methods Sample}

This cross-sectional study was conducted on a convenience sample of the first 74 outpatients affected by schizophrenia or schizoaffective disorders according to DSM-IV-TR, who attended a University-based community mental health centre $(\mathrm{CMHC})$ and had previously concluded a two-year follow up period in the above cited observational study [14]. Patients with comorbid psychiatric and/or physical disorders were included in the study, with the exception of those affected by mental retardation or severe organic brain diseases. Subjects underwent standard care routinely available in Italian CMHCs (clinical monitoring at least on a monthly basis; pharmacological treatment; home care when required, psychosocial and rehabilitation interventions tailored to patient's needs). The study was approved by the Ethics Committee of the Local Health Authorities in Cagliari (Italy) and was conducted in compliance with national laws. All patients gave their informed consent to take part in the study. The sample included patients affected by schizophrenia ( $\mathrm{N}=32,43 \%)$ or schizoaffective disorders $(\mathrm{N}=42,57 \%)$. The sociodemographic characteristics of the sample are reported in Table 1; clinical characteristics are reported in Table 2 . The sample included patients who were mostly male (70\%), with a mean age of 43.5 years, single ( $85 \%$ ), unemployed (77\%), and with a good level of education (mean years of education $=11$ ). Patients were characterized by early age at onset of the illness (approx. 23 years), a long clinical history (mean duration approx. 15 years), and a continuous course of the disorder (approx. $57 \%$ of cases); all patients had been prescribed psychopharmacological treatment.
Table 1 Sociodemographic characteristics of the sample $(\mathbf{N}=74)$

\begin{tabular}{ll}
\hline Characteristics & Statistics \\
\hline Gender N (\%) & \\
Males & $52(70.3)$ \\
Females & $22(29.7)$ \\
Age & \\
Mean \pm SD & $43.46(10.06)$ \\
Range & $25-68$ \\
Education & \\
Mean years \pm SD & $10.67(3.88)$ \\
Range & $4-14$ \\
Marital status N (\%) & \\
Singles & $63(85.1)$ \\
Married & $11(14.9)$ \\
Occupation N (\%) & \\
Employed & $17(23)$ \\
Unemployed & $57(77)$ \\
\hline
\end{tabular}

\section{Ratings}

Evaluation was performed by residents in psychiatry using a set of standardized methods, after adequate training in the use of all adopted instruments. Personal and social data, and clinical history were collected in a structured interview purpose-developed for the study. After providing informed consent, patients were interviewed using the Italian versions [15, 16] of SCID-I [17] and SCID-II [18]; inter-rater reliability, assessed using Cohen's K before the study, was higher than 0.80. Symptom severity was evaluated using the Italian version [19] of PANSS [20]' consisting of 30 items grouped into three distinct symptom clusters (positive, negative, general psychopathology) rated on a 7 point scale; an accurate picture of symptomatology is generally based on summary score obtained for each symptom cluster and on total score. Interviews were conducted by means of the Italian version [21] of the Structured Clinical Interview for Positive and Negative Scale (SCI-PANSS) [22]. Ratings were based on criteria indicated in the PANSS Manual [23] and, wherever possible, PANSS assessment included a standard section of queries addressed to treating clinicians and caregivers. The global clinical status was also evaluated by CGI-SCH [24], the version of the CGI (clinical global impression scale) adapted for use in Schizophrenia; the CGI-SCH provides for the global assessment of positive, negative, cognitive, and depressive symptoms and overall clinical condition over the week before the visit; CGI takes three aspects into account: severity of symptoms, global improvement and an efficacy index. For the purposes of this study, only the severity scale (CGI$\mathrm{SCHs}$ ) was used; scores ranged from 1 (no symptoms) 
Table 2 Clinical characteristics of the sample

\begin{tabular}{|c|c|}
\hline Characteristics & Statistics \\
\hline \multicolumn{2}{|l|}{ Diagnosis N (\%) } \\
\hline Schizophrenia & $32(43.2)$ \\
\hline Schizoaffective disorders & $42(56.8)$ \\
\hline \multicolumn{2}{|l|}{ Age at onset } \\
\hline Mean $\pm S D$ & $22.83(10.06)$ \\
\hline Range & $13-53$ \\
\hline \multicolumn{2}{|l|}{ Duration of untreated psychosis } \\
\hline Mean months \pm SD & $26.05(53.85)$ \\
\hline Range & $24-564$ \\
\hline \multicolumn{2}{|l|}{ Comorbidities N (\%) } \\
\hline Anxiety disorders & $7(9.5)$ \\
\hline Personality disorders & $8(11.0)$ \\
\hline Alcohol abuse/dep & $9(12.3)$ \\
\hline Other subst abuse/dep & $16(21.9)$ \\
\hline \multicolumn{2}{|l|}{ Previous clinical course N (\%) } \\
\hline Episodic with remission & $11(14.9)$ \\
\hline Episodic without remission & $17(23.0)$ \\
\hline Continuous & $42(56.8)$ \\
\hline Non specified & $4(5.4)$ \\
\hline \multicolumn{2}{|l|}{ Previous hospital adm N (\%) } \\
\hline Yes & $47(63.5)$ \\
\hline No & $27(36.5)$ \\
\hline \multicolumn{2}{|l|}{ Previous suicide attempts N (\%) } \\
\hline Yes & $20(27)$ \\
\hline No & $54(73)$ \\
\hline \multicolumn{2}{|l|}{ Previous legal problems N (\%) } \\
\hline Yes & $6(8.1)$ \\
\hline No & 68 (91.9) \\
\hline \multicolumn{2}{|l|}{ Treatments N (\%) } \\
\hline Antipsychotics & $74(100.0)$ \\
\hline Mood stabilizers & $17(23.0)$ \\
\hline Antidepressants & $19(25.7)$ \\
\hline Anxiolytics & $42(56.7)$ \\
\hline Other & $13(17.6)$ \\
\hline Psychotherapy & $9(12.1)$ \\
\hline Rehabilitation programmes & $16(21.6)$ \\
\hline \multicolumn{2}{|l|}{ Remission N (\%) } \\
\hline Clinical & $40(54.1)$ \\
\hline Functional & $22(29.7)$ \\
\hline Clinical + functional (recovery) & $20(27.0)$ \\
\hline
\end{tabular}

to 7 (most severe symptoms). Cognitive functioning was evaluated by means of BACS [25], a scale comprising a series of cognitive dimensions, namely list learning, digit sequencing, category instances and controlled oral word association test, symbol coding and executive functions; a gender/age/education adjusted score, and subsequently an equivalent score were calculated [26]. MMSE [27] was also administered, calculating an age/education adjusted score [28]. Functioning was evaluated by PSP [8], assumed as a gold standard, to assess social functioning of patients in four main areas: socially useful activities, personal and social relationships, self-care and disturbing/aggressive behaviours. Each area was evaluated on a six-point scale, where lower ratings indicate better functioning. A comprehensive overall score ranging from 1 (maximum dysfunction) to 100 (maximum functioning) was attributed, based on scores obtained at each single area. A total score exceeding 70 indicates a condition of "functional remission", with scores related to overall good functioning. A non-standardized interview was conducted with the patient, caregivers (when available) and with the treating physician, with the aim to assess functioning by means of PSP. Patients were also submitted to the Subjective Well-being under Neuroleptic scale (SWN-S) [29], a self-administered rating scale devoted to evaluating the psychological and physical well-being of patients treated with neuroleptics. For the purpose of the study the short version (20 items, including 5 subscales: mental functions, self-control, physical function, emotional control, social capability) was used. The Mini-ICFAPP $[10,11]$ is a clinician-rated instrument developed to measure limitations of capacity in people with mental disorders; these limitations are rated in terms of competence throughout thirteen domains, namely adherence to regulations, planning and structuring of tasks, flexibility, competency, endurance, assertiveness, contact with others, group integration, intimate relationships, non-work activities, self-care, mobility and competence to judge and decide. Each dimension is rated on a five-point Likert scale ( $0=$ no impairment; $5=$ total disability). Ratings are based on information provided by patients, carers, clinical records, as well as direct observation of subjects and on anchor definitions of each item described in the Italian version of the Manual [30].

Inter-rater reliability of PANSS evaluations in terms of ICC (Intraclass correlation coefficient) ranged from 0.65-0.95 for the PANSS total score, from 0.72-0.97 or the CGI-SGH total score, from $0.79-0.94$ for the PSP total score and from $0.81-0.93$ for Mini-ICF-APP total score.

\section{Validity}

Concurrent validity was evaluated calculating the correlations between scores at Mini-ICF-APP and PSP, the latter being considered the standard of reference for evaluation of personal and social functioning; moreover, correlations of scores obtained at Mini-ICF-APP and PANSS, CGI-SCHs, BACS, MMSE were calculated, based on the hypothesis of an existing, significant correlation between results relating to symptoms and cognitive status and 
functioning (i.e. the lower the scores obtained at clinical and cognitive scales, the lower the score at MiniICF-APP). Construct validity was evaluated by means of known-groups technique, which involves administering the measurement instrument to groups expected to differ due to known characteristics. Thus Mini-ICF-APP was evaluated by comparing differences in mean scores at each single item and total score obtained by "remitted" and "non remitted" patients and "recovered" and "unrecovered" subjects at the time of evaluation (24 months). The working hypothesis was based on the assumption that both remission and recovery should be associated with achievement of better results at Mini-ICF-APP, i.e. lower mean scores. Clinical remission was assessed using the schizophrenia remission working group criteria (SRWG-cr) [30], based on ratings at eight focal symptoms in positive, negative and general psychopathology subscales of PANSS (P1, P2, P3, N1, N4, N6, G5, G9); patients were judged to be in clinical remission according to a severity criterion (scores obtained at each of these items were required to be $\leq 3$ points, indicating mild severity of symptoms). Due to the cross-sectional nature of the study, clinical remission was evaluated on the basis of severity criterion alone, excluding the duration criterion (remission maintained for 6-months). With regard to functional remission, patients with a PSP total score $>70$, i.e. cut-off score indicating subjects devoid of significantly impaired functioning [8], were deemed to be remitted. Patients' overall clinical status was taken into account, and assessments were carried out to determine whether or not they could be considered as "recovered"; for this purpose, due to the lack of a univocal definition for recovery [31, 32], patients characterized by both clini$\mathrm{cal}$ and functional remission were taken as being recovered. Discriminant validity was evaluated calculating the correlations between scores at Mini-ICF-APP and SWN$\mathrm{S}$, a scale specifically devoted to evaluate subjective-well being of patients undergoing antipsychotic treatment, on the basis of the hypothesis that no significant correlation should be found between results relating to functioning and treatment-related well-being measures.

\section{Statistical analysis}

Categorical data were analyzed using Pearson's $\mathrm{x} 2$ test or Fisher's exact test; continuous variables were assessed by means of Student's " $t$ " test for independent samples. The magnitude of differences in mean scores obtained at different rating scales used in the study was calculated by means of Cohen's " $d$ ". Correlations between continuous variables were calculated the Pearson' $r$ coefficient. Data analyses were performed using SPSS 19.0. Level of significance was set at a $p$ value $\leq 0.05$ for two-tailed hypothesis.

\section{Results}

Ratings at PANSS, CGI-SCHs, BACS, MMSE and PSP are reported in Table 3. Mean scores obtained at clinical and cognitive scales show low-moderate levels of severity and impairment, as expected in a cohort of relatively stable patients in a community treatment setting. No significant gender or diagnosis differences were detected for sociodemographic and clinical variables. The median score at Mini-ICF-APP is generally comprised between 1 and 2 for the majority of items, thus indicating a low-moderate level of impairment (Table 4); no differences were found in mean scores according to gender and diagnosis. With regard to correlational analyses, Mini-ICG-APP total score displayed a significant correlation with PSP total score and scores at the PSP subscales "socially useful activities", "personal and social relationship" and "selfcare"; all Mini-ICF-APP items correlated significantly with PSP total score, with the exception of the "Mobility" item; 4 out of 13 items of Mini-ICF-APP (Adherence to regulations, Structuring of tasks, Flexibility, Self-Care) featured a significant correlation with scores obtained at the PSP subscales "socially useful activities", "personal and social relationship" and "self-care", while 8 out of 13 items of Mini-ICF-APP (Competency, Endurance, Assertiveness, Contact with Others, Group Integration, Intimate Relations, Spontaneous activity, Judgement) correlated significantly with 2 out of 4 PSP subscales, namely "Socially Useful Activities", "Personal and Social Relationship"; only one Mini-ICF-APP item (mobility) was devoid of any correlation with PSP subscales (Table 5). In the symptomatology domain, Mini-ICF-APP total score significantly correlated with scores at CGI$\mathrm{SCH}$ s cognitive and depressive subscales and with the overall scale; moreover, a correlation was observed with PANSS total score and negative and general psychopathology scales (Table 6). With regard to cognition, MiniICF-APP total score significantly correlated with MMSE score, and with scores at BACS scales "Verbal memory", "Verbal fluency" and "Tower of London" (Table 7).

On assessing construct validity, total mean scores of clinically remitted patients on Mini-ICF-APP were found to be significantly higher than those of unremitted patients; moreover, clinically remitted patients obtained significantly higher mean scores at 10 out of 13 MiniICF-APP items (Table 8). Similarly, total mean scores obtained by functionally remitted subjects at Mini-ICFAPP were significantly higher than those of unremitted patients, with significantly higher mean scores being attained in 9 out of 13 items compared to functionally unremitted patients (Table 9). Mean total Mini-ICFAPP scores rated by recovered patients were significantly higher than those of unrecovered subjects, with similar results being registered in 4 out of 13 items of the scale 
Table 3 Rating scales mean scores

\begin{tabular}{|c|c|c|c|}
\hline Rating scale & Mean value (SD) & Median & Range \\
\hline CGI SCHs positive symptoms & $2.27(1.43)$ & 2.00 & $1-6$ \\
\hline CGI SCHs negative symptoms & $2.78(1.40)$ & 3.00 & $1-6$ \\
\hline CGI SCHs depressive symptoms & $2.20(1.08)$ & 2.00 & $1-6$ \\
\hline CGI SCHs cognitive symptoms & $2.69(1.43)$ & 3.00 & $1-7$ \\
\hline CGI SCHs total score & $3.12(1.22)$ & 3.00 & $1-6$ \\
\hline PANSS positive & $10.36(3.60)$ & 9.00 & $7-25$ \\
\hline PANSS negative & $14.51(6.93)$ & 12.50 & $7-36$ \\
\hline PANSS general psychopathology & $26.51(8.84)$ & 23.00 & $16-47$ \\
\hline PANSS total score & $51.39(17.0)$ & 47.00 & $30-91$ \\
\hline MMSE & $26.04(3.90)$ & 27.00 & $11-30$ \\
\hline BACS list learning & $14.57(11.80)$ & 11.57 & $2.75-53.25$ \\
\hline BACS digit sequencing task & $11.26(5.88)$ & 11.17 & $0.25-28.25$ \\
\hline BACS verbal fluency/category istances & $10.87(5.24)$ & 10.00 & $1.00-23.25$ \\
\hline BACS semantic fluency/controlled oral word test & $15.38(5.65)$ & 14.50 & $1.00-34.25$ \\
\hline BACS symbol coding & $26.13(13.57)$ & 23.25 & $0.75-65.75$ \\
\hline BACS tower of London & $9.36(7.15)$ & 6.25 & $0-22.50$ \\
\hline PSP socially useful activities & $2.54(1.32)$ & 3.00 & $0-5$ \\
\hline PSP person soc relat & $2.39(1.22)$ & 2.00 & $0-5$ \\
\hline PSP selfcare & $0.81(1.1)$ & 0.00 & $0-4$ \\
\hline PSP dist aggress behav & $0.39(0.79)$ & 0.00 & $0-3$ \\
\hline PSP total & $55.65(16.24)$ & 60.00 & 18-90 \\
\hline
\end{tabular}

CGI-SCHs clinical global impression schizophrenia severity; PANSS positive and negative symptoms scale; BACS brief assessment cognition scale; MMSE mini mental state examination; PSP personal and social performance scale

Table 4 Mini-ICF-APP mean/median scores

\begin{tabular}{lccc}
\hline Item & Mean value (SD) & Median & Range \\
\hline Adherence to regulations & $0.89(1.07)$ & 1.00 & $0-4$ \\
Structuring of tasks & $1.57(1.23)$ & 1.00 & $0-4$ \\
Flexibility & $1.99(1.14)$ & 2.00 & $0-4$ \\
Competency & $2.01(1.20)$ & 2.00 & $0-4$ \\
Endurance & $1.89(1.34)$ & 2.00 & $0-4$ \\
Assertiveness & $1.50(1.21)$ & 1.00 & $0-4$ \\
Contact with others & $1.62(1.22)$ & 1.50 & $0-4$ \\
Group integration & $1.62(1.27)$ & 2.00 & $0-4$ \\
Intimate relations & $1.89(1.25)$ & 2.00 & $0-4$ \\
Spontaneous activity & $1.78(1.17)$ & 2.00 & $0-4$ \\
Self-care & $0.70(0.84)$ & 0.50 & $0-3$ \\
Mobility & $0.85(1.15)$ & 0.00 & $0-4$ \\
Judgement & $1.74(1.12)$ & 2.00 & $0-4$ \\
Total score & $20.07(11.77)$ & 18.50 & $0-46$ \\
\hline
\end{tabular}

(Table 10). Finally, no significant correlations were found between scores at Mini-ICF-APP and SWN-S scales with the only exception of the ICF "persistence" scale, showing a significant negative correlation with SWN-S Self-control $(\mathrm{r}=-0.361, \mathrm{p}<0.01)$, Physical Functioning scales $(\mathrm{r}=-0.301, \mathrm{p}<0.01)$ and with SWN-total score $(\mathrm{r}=-0.305, \mathrm{p}<0.01)$.

\section{Discussion}

The patient sample studied provided an adequate representation of chronic patients with schizophrenia and related disorders generally attending community mental health centres in Italy. Indeed, the sample was made up prevalently by middle-aged, single, unemployed, fairly well educated males, with a long duration of illness, early age at onset, and continuous course of the disorder; ratings obtained at CGI-SCH, PANSS, MMSE, BACS and MMSE, relating to symptoms, cognition and functioning, denote a fairly stable clinical condition characterized by a low/moderate symptoms' severity, cognitive impairment and social disability, as expected in a cohort of relatively stable, chronic patients in a community setting. Indeed, approximately $50 \%$ of our patients were in clinical remission, slightly less than one-third in functional remission, and about one quarter viewed as recovered in line with the adopted criteria, a finding largely consistent with previous data published by our group [14, 33] and with literature findings [34-37]. Mean and median scores obtained at Mini-ICF-APP were generally comprised between 1 and 2 for the majority of items, thus indicating 
Table 5 Correlations between Mini-ICF-APP and PSP scores

\begin{tabular}{|c|c|c|c|c|c|}
\hline & $\begin{array}{l}\text { PSP socially } \\
\text { useful activities }\end{array}$ & $\begin{array}{l}\text { PSP personal } \\
\text { and social relationships }\end{array}$ & PSP selfcare & $\begin{array}{l}\text { PSP aggressive and } \\
\text { disturbing behaviours }\end{array}$ & PSP total score \\
\hline $\begin{array}{l}\text { Mini ICF adherence to } \\
\text { regulations }\end{array}$ & $0.342^{* *}$ & $0.305^{* *}$ & $0.303^{* *}$ & 0.181 & $-0.426^{* *}$ \\
\hline Mini ICF structuring of tasks & $0.305^{* *}$ & $0.414^{* *}$ & $0.268^{*}$ & 0.205 & $-0.389^{* *}$ \\
\hline Mini ICF flexibility & $0.367^{* *}$ & $0.406^{* *}$ & $0.242^{*}$ & 0.112 & $-0.383^{* *}$ \\
\hline Mini ICF competency & $0.460^{* *}$ & $0.360^{* *}$ & 0.182 & 0.038 & $-0.452^{* *}$ \\
\hline Mini ICF judgement & $0.352^{* *}$ & $0.393^{* *}$ & 0.117 & 0.146 & $-0.355^{* *}$ \\
\hline Mini ICF endurance & $0.496^{* *}$ & $0.385^{* *}$ & 0.109 & 0.105 & $-0.459^{* *}$ \\
\hline Mini ICF assertiveness & $0.402^{* *}$ & $0.403^{* *}$ & 0.157 & 0.036 & $-0.376^{* *}$ \\
\hline Mini ICF contact with others & $0.339^{* *}$ & $0.447^{* *}$ & 0.162 & 0.141 & $-0.394^{* *}$ \\
\hline Mini ICF group integration & $0.433^{* *}$ & $0.423^{* *}$ & 0.147 & 0.123 & $-0.417^{* *}$ \\
\hline Mini ICF intimate relations & $0.368^{* *}$ & $0.459^{* *}$ & 0.178 & 0.169 & $-0.481^{* *}$ \\
\hline $\begin{array}{l}\text { Mini ICF spontaneous } \\
\text { activity }\end{array}$ & $0.331^{* *}$ & $0.384^{* *}$ & 0.183 & 0.196 & $-0.474^{* *}$ \\
\hline Mini ICF self-care & $0.417^{* *}$ & $0.408^{* *}$ & $0.314^{* *}$ & 0.137 & $-0.465^{* *}$ \\
\hline Mini ICF mobility & 0.152 & 0.139 & 0.120 & -0.055 & -0.163 \\
\hline Mini ICF total score & $0.475^{* *}$ & $0.491^{* *}$ & $0.241^{*}$ & 0.152 & $-0.520^{* *}$ \\
\hline
\end{tabular}

${ }^{*} p<0.05,{ }^{* *} p<0.01$

Table 6 Correlations between Mini-ICF-APP total score, CGI-SCH and PANSS ratings

\begin{tabular}{lllllll}
\hline $\begin{array}{l}\text { CGI-SCH } \\
\text { positive } \\
\text { symptoms }\end{array}$ & $\begin{array}{l}\text { CGI-SCH } \\
\text { negative } \\
\text { symptoms }\end{array}$ & $\begin{array}{l}\text { CGI-SCH } \\
\text { depressive } \\
\text { symptoms }\end{array}$ & $\begin{array}{l}\text { CGI-SCH } \\
\text { cognitive } \\
\text { symptoms }\end{array}$ & $\begin{array}{l}\text { CGI-SCH } \\
\text { total } \\
\text { score }\end{array}$ & $\begin{array}{l}\text { PANSS } \\
\text { positive } \\
\text { scale }\end{array}$ & $\begin{array}{l}\text { PANSS } \\
\text { negative } \\
\text { scale }\end{array}$ \\
\hline 0.062 & $0.305^{* *}$ & $0.325^{* *}$ & $0.248^{*}$ & $0.455^{* *}$ & 0.189 & $\begin{array}{l}\text { PANSS total } \\
\text { psycho } \\
\text { pathology } \\
\text { scale }\end{array}$ \\
\hline
\end{tabular}

${ }^{*} p=0.05,{ }^{* *} p=0.01$

Table 7 Correlations between Mini-ICF-APP total scores, MMSE and BACS

\begin{tabular}{llllll}
\hline MMSE & $\begin{array}{l}\text { BACS list } \\
\text { learning }\end{array}$ & $\begin{array}{l}\text { BACS digit } \\
\text { sequencing } \\
\text { task }\end{array}$ & $\begin{array}{l}\text { BACS verbal } \\
\text { fluency/category } \\
\text { instances }\end{array}$ & $\begin{array}{l}\text { BACS semantic } \\
\text { fluency/controlled } \\
\text { oral word test }\end{array}$ & $\begin{array}{l}\text { BACS symbol } \\
\text { coding }\end{array}$ \\
\hline$-0.260^{*}$ & $-0.237^{*}$ & 0.215 & $-0.390^{* *}$ & -0.225 & -0.146 \\
of London
\end{tabular}

${ }^{*} \mathrm{p}<0.05,{ }^{* *} \mathrm{p}<0.01$

a low-moderate level of impairment, with only two items (self-care and mobility) showing very low scores (both means and medians lower than one), indicating the absence of, or minimal impairments in these areas; this finding was very similar to that of Balestrieri et al. in their validation study of the Italian version of the Mini-ICFAPP [13]. In our sample, Mini-ICF-APP mean total score was approx. 20, somewhat lower than that observed in the subsample of schizophrenic subjects (mean score approx. 30) in the above cited study [13], and higher than that observed by Molodynski et al. [12] (mean score approx. 14) in a subsample of schizophrenic patients included in the validation study for the English version of the instrument. Bearing in mind that our sample was constituted by a cohort of clinically chronic outpatients who were all on antipsychotic treatment, the somewhat low level of disability reflected by the mean total score at Mini-ICFAPP is not surprising, and is consistent with the English study, which similarly comprised schizophrenic outpatients in attending two community mental health centres, featuring a low-moderate level of severity of symptomatology and functioning, as reflected respectively by mean scores at BPRS (approx. 38) and PSP (approx. 53). On the contrary, the study conducted by Balestrieri et al. 
Table 8 Mini-ICF-APP mean scores \pm SD according to clinical remission status

\begin{tabular}{|c|c|c|c|c|c|}
\hline Item & Remitted $(\mathrm{N}=40)$ & Non remitted $(\mathrm{N}=34)$ & Cohen's d & t value (df) & $p$ value \\
\hline Adherence to regulations & $0.70(0.99)$ & $1.12(1.12)$ & -0.379 & $-1.682(66.5)$ & 0.094 \\
\hline Structuring of tasks & $1.15(1.23)$ & $2.06(1.04)$ & -0.798 & $-3.438(72)$ & 0.001 \\
\hline Flexibility & $1.67(1.22)$ & $2.35(0.92)$ & -0.629 & $-2.651(72)$ & 0.010 \\
\hline Competency & $1.75(1.22)$ & $2.32(1.09)$ & -0.492 & $-2.188(72.00)$ & 0.038 \\
\hline Endurance & $1.57(1.30)$ & $2.26(1.31)$ & -0.528 & $-2.266(69.89)$ & 0.027 \\
\hline Assertiveness & $1.32(1.16)$ & $1.71(1.24)$ & -0.324 & $-1.352(68.34)$ & 0.181 \\
\hline Contact with others & $1.32(1.18)$ & $1.97(1.19)$ & -0.506 & $-2.327(69.90)$ & 0.023 \\
\hline Group integration & $1.22(1.25)$ & $2.09(1.14)$ & -0.727 & $-3.107(71.64)$ & 0.003 \\
\hline Intimate relations & $1.50(1.21)$ & $2.35(1.12)$ & -0.729 & $-3.127(72)$ & 0.003 \\
\hline Spontaneous activity & $1.37(1.17)$ & $2.26(0.99)$ & -0.821 & $-3.537(72)$ & 0.001 \\
\hline Self-care & $0.55(0.68)$ & $0.88(0.98)$ & -0.391 & $-1.671(57.36)$ & 0.10 \\
\hline Mobility & $0.82(1.15)$ & $0.88(1.17)$ & -0.050 & $-0.211(69.40)$ & 0.83 \\
\hline Judgement & $1.50(1.15)$ & $2.03(1.03)$ & -0.485 & $-2.085(71.82)$ & 0.041 \\
\hline Total score & $16.47(11.84)$ & $24.29(10.33)$ & -0.703 & $-3.033(71.94)$ & 0.003 \\
\hline
\end{tabular}

Table 9 Mini-ICF-APP mean scores \pm SD according to functional remission status

\begin{tabular}{|c|c|c|c|c|c|}
\hline Item & Remitted ( $N=22)$ & Non remitted $(\mathrm{N}=52)$ & Cohen's d & t value (df) & $p$ value \\
\hline Adherence to regulations & $0.54(0.80)$ & $1.04(1.13)$ & -0.510 & $-2.122(55.5)$ & 0.038 \\
\hline Structuring of tasks & $1.18(1.33)$ & $1.73(1.16)$ & -0.440 & $-1.683(35.06)$ & 0.101 \\
\hline Flexibility & $1.54(1.22)$ & $2.17(1.06)$ & -0.551 & $-2.095(35.03)$ & 0.043 \\
\hline Competency & $1.64(1.40)$ & $2.17(1.07)$ & -0.425 & $-1.608(32.08)$ & 0.118 \\
\hline Endurance & $1.18(1.29)$ & $2.19(1.25)$ & -0.795 & $-3.096(38.42)$ & 0.004 \\
\hline Assertiveness & $1.23(1.23)$ & $1.61(1.19)$ & -0.314 & $-1.251(38.41)$ & 0.218 \\
\hline Contact with others & $1.23(1.30)$ & $1.79(1.16)$ & -0.454 & $-1.744(35.71)$ & 0.090 \\
\hline Group integration & $1.14(1.21)$ & $1.83(1.25)$ & -0.560 & $-2.227(40.85)$ & 0.032 \\
\hline Intimate relations & $1.23(1.23)$ & $2.17(1.15)$ & -0.789 & $-3.078(37.25)$ & 0.004 \\
\hline Spontaneous activity & $1.32(1.29)$ & $1.98(1.07)$ & -0.556 & $-2.122(34.02)$ & 0.041 \\
\hline Self-care & $0.41(0.66)$ & $0.83(0.87)$ & -0.543 & $-2.232(51.79)$ & 0.030 \\
\hline Mobility & $0.82(1.33)$ & $0.86(1.08)$ & -0.030 & $-0.147(33.36)$ & 0.884 \\
\hline Judgement & $1.41(1.18)$ & $1.88(1.08)$ & -0.415 & $-1.623(36.54)$ & 0.113 \\
\hline Total score & $14.86(12.02)$ & $22.27(11.05)$ & -0.641 & $-2.479(36.76)$ & 0.018 \\
\hline
\end{tabular}

[13] included a subsample of schizophrenic patients in the care of a CMHC who were characterized by a more severe clinical status (median CGIs $=6$; BPRS median score 75 ), and of which $40 \%$ were not taking any form of psychopharmacological treatment; this finding may explain, at least in part, the higher mean total score at Mini-ICF-APP observed in this study. With regard to concurrent validity, a high negative correlation was found between mean total score at Mini-ICF-APP (where lower scores indicate better functioning) and mean total score at PSP (where lower scores indicate worse functioning); moreover, a significant negative correlation was found between all Mini-ICF-APP items, with the exception of "mobility", and mean total score at PSP. When taking into account correlations between each item of Mini-ICFAPP and PSP domains, we found that four items of the former ("adherence to regulations", "structuring of tasks", "flexibility", and "spontaneous activity") were significantly correlated with three PSP domains ("socially useful activities", "personal and social relations" and "self-care"). Furthermore, eight items of Mini-ICF-APP ("flexibility", "competency", "endurance", "assertiveness", "contact with others", "group integration", "intimate relationships" "spontaneous activities" and "judgement") significantly correlated with two PSP domains ("socially useful activities" and "personal and social relations"). The only item 
Table 10 Mini-ICF-APP mean scores \pm SD according to recovery status

\begin{tabular}{|c|c|c|c|c|c|}
\hline Item & Recovered $(\mathrm{N}=20)$ & Non recovered $(N=54)$ & Cohen's d & t value (df) & $p$ value \\
\hline Adherence to regulations & $0.55(0.82)$ & $1.02(1.12)$ & -0.478 & $-1.954(46.24)$ & 0.057 \\
\hline Structuring of tasks & $1.15(1.38)$ & $1.72(1.14)$ & -0.450 & $-1.650(29.04)$ & 0.110 \\
\hline Flexibility & $1.50(1.23)$ & $2.16(1.06)$ & -0.574 & $-2.139(29.96)$ & 0.041 \\
\hline Competency & $1.65(1.46)$ & $2.15(1.07)$ & -0.390 & $-1.604(72)$ & 0.113 \\
\hline Endurance & $1.30(1.30)$ & $2.11(1.29)$ & -0.625 & $-2.382(33.92)$ & 0.023 \\
\hline Assertiveness & $1.30(1.26)$ & $1.57(1.19)$ & -0.220 & $-0.843(32.37)$ & 0.406 \\
\hline Contact with others & $1.30(1.34)$ & $1.74(1.17)$ & -0.349 & $-1.298(30.32)$ & 0.204 \\
\hline Group integration & $1.10(1.25)$ & $1.81(1.23)$ & -0.572 & $-2.191(33.46)$ & 0.036 \\
\hline Intimate relations & $1.30(1.26)$ & $2.11(1.18)$ & -0.663 & $-2.502(32.03)$ & 0.018 \\
\hline Spontaneous activity & $1.30(1.34)$ & $1.96(1.06)$ & -0.546 & $-1.990(28.32)$ & 0.056 \\
\hline Self-care & $0.45(0.68)$ & $0.79(0.87)$ & -0.435 & $-1.781(43.25)$ & 0.082 \\
\hline Mobility & $0.90(1.37)$ & $0.83(1.07)$ & 0.056 & $-0.196(28.13)$ & 0.846 \\
\hline Judgement & $1.40(1.23)$ & $1.87(1.06)$ & -0.409 & $-1.512(30.16)$ & 0.141 \\
\hline Total score & $15.20(12.46)$ & $21.87(11.08)$ & -0.565 & $-2.105(30.82)$ & 0.044 \\
\hline
\end{tabular}

of Mini-ICF-APP that failed to show a significant correlation with any PSP domain was "Mobility", while the PSP domain that failed to correlate with any Mini-ICFAPP item was "aggressive and disturbing behaviour". Taken together, these findings demonstrate a fairly good concurrent validity with PSP, as previously shown in the validation study for both the Italian [13] and the English versions [12] of the instrument. It should be pointed out that similar results were also obtained by Schaub et al. [38], who found a highly significant correlation between Mini-ICF-APP total score and PSP total score, in a study performed to validate the German version of the instrument in chronic schizophrenic patients.

Although schizophrenic symptomatology and functioning are only partially inter-related, being seen as semiindependent outcome measures [39], and despite the fact that clinical remission does not always imply functional remission [33, 35], significant positive correlations between symptom severity and impaired functioning are generally reported in the literature [1]. In our study, Mini-ICF-APP total scores were significantly correlated with overall scores of both CGI-SCHs and PANSS, a finding indicating a clear relationship between severity of symptomatology and degree of severity of functional deficits measured by Mini-ICF-APP, while no correlations were observed with ratings of positive symptoms evaluated by both CGI-SCHs and PANSS. Lastly, depressive symptomatology and cognitive impairment evaluated by CGI-SCHs, and general psychopathology evaluated by means of PANSS, were all significantly and positively correlated with Mini-ICF-APP total scores. These results are largely in agreement with those of Balestrieri et al. [13]' who found a highly significant correlation between
Mini-ICF-APP total score and both CGI-severity score and BPRS global score in their subsample of schizophrenics. Moreover, using BPRS, the same authors found a significant correlation between Mini-ICF-APP total score and several "positive" symptoms ("grandiosity", "hostility", "suspiciousness", "hallucinations", "unusual thought content"), "mood" symptoms ("guilt", "elated mood", "excitement"), and "negative symptoms" ("emotional withdrawal", "uncooperativeness"). The partial incongruence between the results obtained in our study and those of the study mentioned above [13]' particularly the association between positive symptoms and functioning, may at least in part be explained by the previously cited difference in severity of clinical status between patient samples investigated in the two studies, as the study conducted by our group comprised a fairly stable sample of patients with less severe chronic illness. It should be taken into account that contrasting data are present in literature relating to the role of positive symptoms in disability, while a positive correlation of functioning with negative and depressive symptoms is generally reported $[5,38]$. In addition to the correlation observed between severity of cognitive deficits clinically measured using CGI-SCHs cognitive scale and severity of functioning measured by Mini-ICFAPP, we also detected significant correlations with the MMSE and with three out of six BACS domains; this finding was not unexpected, particularly in view of the well established relationship between cognitive and real-world functioning deficits in schizophrenic patients [40]. To establish construct validity, differences in Mini-ICF-APP scores were evaluated, resulting in the expected outcome of significant differences between remitted and recovered patients. Indeed, both clinically and functionally remitted 
patients displayed a significantly higher Mini-ICF-APP total score compared to unremitted subjects, thus indicating consistently improved functioning; a similar finding was revealed for "recovered" patients, i.e. patients characterized by both clinical and functional remission. Moreover, on analysis, differences in mean scores obtained at each of the thirteen Mini-ICF-APP items provided confirmation of the above findings for a consistent number of items, highlighting better results among remitted and recovered patients for the majority of functioning aspects covered by Mini-ICF-APP. Finally, as expected we found no consistent correlations between Mini-ICF-APP and SWN-S, a result in favour of a fairly good discriminant validity of the instrument.

Before drawing conclusions, several limitations of the present study should be taken into account. In particular, the rather limited size of the study sample, and the prevalent focus on chronic outpatients limit the validity of our findings to the sole patients studied. Additionally, sample heterogeneity should be taken into account, due to inclusion in the study of patients affected by both schizophrenia and schizoaffective disorders. However, it should be underlined that in our cohort no differences in sociodemographic and clinical parameters were detected between the two diagnostic subsamples. However, even in the light of these limitations, the evidence obtained would seem to be of interest for clinicians.

\section{Conclusions}

Results obtained in this study show that the Mini-ICFAPP is a valid measure of functioning for use in chronic patients affected by schizophrenia and schizoaffective disorders. Moreover, the tool holds characteristics which are not available in other instruments [13], which specifically enable the measurement of "capacities", and addresses aspects of functioning of particular relevance to the working environment [12]. Therefore, in our opinion, Mini-ICF-APP should be given due recognition and added to the scenario of existing instruments intended for use in evaluating functioning in the mentally ill. Moreover, the user-friendly and streamlined application of the instrument is particularly suited for use in a routine psychiatric setting.

\footnotetext{
Authors' contributions

FP and AF contributed to study conception and design, statistical analysis and interpretation of data and drafting the manuscript; MT and BG gave a substantial contribution to data acquisition, data base-management and analysis of the data; $\mathrm{BC}$ contributed to study conception and design, analysis and interpretation of data and to revise the paper. All authors read and approved the final manuscript.
}

\section{Author details}

${ }^{1}$ Department of Public Health, Clinical and Molecular Medicine-Unit of Psychiatry, University of Cagliari, Via Liguria 13, 0917 Cagliari, Italy. ${ }^{2}$ Department of Psychiatry, University of Naples SUN, Naples, Italy.

\section{Acknowledgements}

The authors thanks the other members of the Cagliari Recovery Study Group: Davide Aru, Chiara Bandecchi, Elena Corda, Francesca Fatteri, Alice Ghiani, Alice Lai, Serena Lai, Lorena Lai, Tiziana Lepori, Raffaella Maccioni, Paola Milia, Sonia Pintore, Silvia Pirarba, Elisabetta Piras, Sara Piras, Laura Puddu, Elisabetta Sarritzu, Manuela Taberlet, Cristina Tocco, Enrico Zaccheddu, for their contribution to the study and Ms Anne Farmer for having revised the English version of the paper.

\section{Competing interests}

The authors declare that they have no competing interests.

Received: 23 June 2015 Accepted: 21 September 2015

Published online: 31 October 2015

\section{References}

1. Carpiniello B, Carta MG. Disability in schizophrenia. Intrinsic factors and prediction of psychosocial outcome. An analysis of literature. Epidemiol Psych Soc. 2002;11:45-59.

2. National Institute for Clinical Excellence. Core interventions in the treatment and management of schizophrenia in primary and secondary care: clinical guideline. London: NICE; 2002.

3. Nasrallah HA, Lasser R. Improving patients' outcomes in schizophrenia: achieving remission. J Psychopharmacol. 2006;20(6 Suppl):57-61.

4. Lysaker PA, Roe D, Buck KD. Recovery and wellness amidst schizophrenia: definitions, evidence, and the implications for clinical practice. J Am Psych Nurs Ass. 2010;16:36-42.

5. Carpiniello B. Social functioning and schizophrenia. Giorn It Psicopatol. 2010;16:227-30.

6. American Psychiatric Association. The global assessment of functioning, in DSM-IV, diagnostic and statistical manual of mental disorders. 4th ed. Washington, DC: American Association Press; 1994.

7. Smith GN, Ehmann TS, Flynn SW, et al. The assessment of symptom severity and functional impairment with DSM-IV axis V. Psychiatr Serv. 2011;62:411-7.

8. Morosini PL, Magliano L, Brambilla L, et al. Development, reliability and acceptability of a new version of the DSM-IV Social and Occupational Functioning Assessment Scale (SOFAS) to assess routine social functioning. Acta Psychiatr Scand. 2000;101:323-9.

9. Ustun TB, Kostanjsek N, Chatterji S, Chatterji S, et al. Measuring health and disability: manual for WHO disability assessment schedule (WHODAS 2.0). Geneva:WHO Press; 2010.

10. Linden M, Baron M. Das Mini-ICF-Rating für Psychische Störungen (MiniICF-P): Ein Kurzinstrument zur Beurteilung von Fähigkeitsstörungen bei psychischen Erktankungen. Rehabilitation. 2005:44:144-51.

11. Baron S, Linden M. Disorders of functioning and disorders of capacity in relation to sick leave in mental disorders. Int J Soc Psychiatry. 2009;55:57-63.

12. Molodynski A, Linden M, Jukel G, et al. The reliability, validity and applicability of an English language version of the Mini-ICG-APP. Soc Psychiatry Psychiatr Epidemiology. 2013;48:1347-54.

13. Balestrieri M, Isola M, Bonn $\mathrm{R}$, et al. Validation of the Italian version of MiniICF-APP, a short instrument fro rating activity and participation restrictions in psychiatric disorders. Epidemiol Psychiatr Sci. 2013;22:81-91.

14. Carpiniello B, Pinna F, Tusconi M, et al. Gender differences in remission and recovery of schizophrenic and schizoaffective patients: preliminary results of prospective cohort study. Schiz Res Treat. 2012;2012:576369. doi:10.1155/2012/576369.

15. First MB, Spitzer RL, Williams JBW, et al. Structured clinical interview for DSM IV Axis I disorders (SCID-I) Versione Italiana a cura di Mazzi F, Morosini P, De Girolamo G, Lusetti M, Guaraldi P. Firenze: Organizzazioni Speciali; 2000

16. First MB, Gibbon M, Spitzer RL, et al. Structured clinical interview for DSM IV Axis II personality disorders - Versione Italiana a cura di Mazzi F, Morosini P, De Girolamo G, Lusetti M, Guaraldi P, Firenze: Organizzazioni Speciali; 2003.

17. First, MB, Spitzer RL, Williams JBW, et al. Structured clinical interview for DSM IV Axis I disorders—research version (SCID-I, version 2. 0). New York: Biometrics Research Department, New York State Psychiatric Institute; 1996. 
18. First MB, Gibbon M, Spitzer RL, et al. Structured clinical interview for DSM IV Axis II personality disorders - research version (SCID II, version 2.0). New York: Biometrics Research Department, New York State Psychiatric Institute; 1996.

19. Pancheri P, Brugnoli R, Carilli L, et al. Valutazione dimensionale della sintomatologia schizofrenica. Validazione della versione italiana della Scala per la valutazione dei Sintomi Positivi e Negativi (PANSS). Giorn Ital Psicopat. 1995;1:60-75.

20. Kay SR, Fiszbein A, Opfer LA. The positive and negative syndrome scale(PANSS) for schizophrenia. Schizophr Bull. 1987;13:261-76.

21. Kay SR, Opler RA, Fiszbein A. The structured clinical interview for positive and negative syndromes of schizophrenia. Versione Italiana a cura di Migone P. Parma: Università di Parma; 1999.

22. Kay SR, Opler RA, Fiszbein A. The structured clinical interview for positive and negative syndromes of schizophrenia. New York: Multi-Health Systems; 1992.

23. Kay SR, Opler RA, Fiszbein A, et al. Positive and negative syndrome scale (PANSS) technical manual. New York: Multi-Health System; 2006.

24. Haro JM, Kamath SA, Ochoa S, et al. The Clinical global impression-schizophrenia scale: a simple instrument to measure the diversity of symptoms present in schizophrenia. Acta Psychiatr Scand. 2003;416(Suppl):16-23.

25. Keefe RS, Goldberg TE, Harvey PD, Gold JM, et al. The brief assessment of cognition in schizophrenia: reliability, sensitivity and comparison with a standardized neurocognitive battery. Schizophr Res. 2004;68:283-97.

26. Anselmetti S, Poletti P, Ermoli E, et al. The brief assessment of cognition in schizophrenia. Normative data for the Italian population. Neurol Sci. 2008;29:85-92.

27. Folstein MF, Folstein SE, McHugh PR. "Mini mental state". A practical method for grading the cognitive state of patients for the clinician. J Psychiatr Res. 1975;12:189-98.

28. Measso G, Cavarzeran F, Zappalà G, et al. The Mini mental state examination. Normative study of an Italian random sample. Develop Neuropsychol. 1993:9:77-85.

29. Naber D, Moritz S, Lambert M, et al. Improvement of schizophrenic patients' subjective well-being under atypical antipsychotic drugs. Schizophr Res. 2001;50:79-88.
30. Linden M, Baron S, Muschalla B. Mini-ICF-APP, manuale. Adattamento Italiano, a cura di Balestrieri M, Maso E. Firenze: Giunti O.S. Organizzazioni Speciali; 2012

31. Andreasen NC, Carpenter WT Jr, Kane JM, et al. Remission in schizophrenia: proposed criteria and rationale for consensus. Am J Psychiat. 2005;162:441-9.

32. Emsley R, Chiliza B, Asmal L, et al. The concepts of remission and recovery in schizophrenia. Curr Opin Psychiatry. 2011;24:114-21.

33. Pinna F, Tusconi $M$, Bosia $M$, et al. Criteria for symptom remission revisited: a study of patients affected by schizophrenia and schizoaffective disorders. BMC Psychiatry. 2013;13:235.

34. Lambert M, Karow A, Leucht S, et al. Remission in Schizophrenia: validity, frequency, predictors and patients' perspectives 5 years later. Dialogues Clin Neurosci. 2010;12:393-407.

35. Karow A, Moritz S, Lambert M, et al. Remitted but still impaired? Symptomatic versus functional remission in patients with schizophrenia. Eur Psychiatry. 2012;27:401-5.

36. Brissos S, Videira Dias V, Balanzà-Martinez V, et al. Symptomatic remission in schizophrenia patients: relationship with social functioning, quality of life, and neurocognitive performance. Schizophr Res. 2011;129:133-6.

37. Turola MC, Comellini G, Galuppi A, Nanni MG, Carantoni E, Scapoli C. Schizophrenia in real life: courses, symptoms and functioning in an Italian Population. Int J Ment Health Syst. 2012;6:22.

38. Schaub C, Brune M, Jaspen E, et al. The illness and everyday living: close interplay of psychopathological syndromes and psychosocial functioning in chronic schizophrenia. Eur Arch Psychiatry Clin Neurosci. 2011;261:85-93

39. Carpenter WT Jr, Strauss JS. The prediction of outcome in schizophrenia IV. Eleven-year follow up of the Washington IPSS cohort. J Nerv Ment Dis. 1991;179:517-25.

40. Green MF, Kern RS, Graff DL, et al. Longitudinal studies of cognition and functional outcome in schizophrenia: implications for MATRICS. Schizophr Res. 2004;26:119-36.

\section{Submit your next manuscript to BioMed Central and take full advantage of:}

- Convenient online submission

- Thorough peer review

- No space constraints or color figure charges

- Immediate publication on acceptance

- Inclusion in PubMed, CAS, Scopus and Google Scholar

- Research which is freely available for redistribution

Submit your manuscript at

www.biomedcentral.com/submit

C Biomed Central 